\title{
Líneas generales de la política externa del Brasil
}

\section{ANTEGEDENTES}

Después de las noticias del viaje de Cristóbal Colón en 1492 al Nuevo Mundo, hubo, sobre todo en la Península Ibérica, intensa movilización diplomática entre Lisboa y Madrid. Por el Tratado de Tordesillas de 1494, se alteraron los límites fijados en el año anterior por la Bula Inter Gaetera, del Papa Alejandro v. Se le atribuyó a Portugal los territorios situados hasta 370 leguas de las islas de Cabo Verde. En 1500 Pedro Alvarez Cabral llega al Brasil. Antes y después de esta fecha, las numerosas tribus indígenas desarrollaron, entre sí y con pueblos europeos, una política externa. Sin embargo, está fuera de los límites de este trabajo efectuar un estudio sobre este particular ${ }^{1}$. En el período de 1580 a 1640 estuvieron unidas las coronas ibéricas, y durante el mismo se amplió considerablemente el territorio ocupado por los lusitanos en América del Sur. Muchas de estas alteraciones limítrofes fueron reconocidas en 1750 por el Tratado de Madrid, en cuya introducción se lee que "cada parte ha de quedarse con lo que actualmente posee". Otros cambios de fronteras fueron establecidos por el Tratado de San IIdefonso, de 1777.

En 1808, el Príncipe Regente Don João, ante la inminencia de la ocupación de Portugal por tropas napoleónicas, se trasladó con la Corte a Río de Janeiro. Brasil fue así el único país americano que hospedó a una familia real europea y fue sede de un imperio. Guando el Rey Don João vI regresó en I821 a Lisboa, permanecieron en el país su hijo Pedro, muchos nobles y numerosos funcionarios vinculados al Servicio Exterior, así como valiosos archivos. En el período de 1808 a 1821 varios gobiernos acreditaron sus represen-

${ }^{1}$ Las relaciones entre las tribus y los portugueses fueron a veces conflictivas, a veces cooperativas. Actualmente los indios en Brasil no llegan a 200.000. La inalienabilidad de las tierras habitadas por ellos está asegurada en el Artf́culo 198 de la Constitución del Brasil, lo que no impide la existencia de diversos problemas con las reservas indfgenas, con respecto a la definición y preservación de sus límites. 
tantes ante la monarquía portuguesa ubicada en Río de Janeiro. EI más antiguo Gonsulado de los Estados Unidos en América, es el establecido en 1808, en la ciudad bahiana de Salvador. En mayo de 1822, José Bonifacio de Andrada y Silva, Ministro de Don Pedro, conocido como patriarca de la Independencia, designó al primer representante del Brasil en el exterior, que fue el Cónsul en Buenos Aires. La Independencia del Brasil fue proclamada por Don Pedio I, nacido en Portugal, que permaneció ell el trono del Imperio del Brasil hasta 1831. A pesar de la gran movilización de tropas, el proceso de emancipación en el Brasil fue comparativamente menos violento y menos demorado que en la América de lengua castellana. Lisboa reconoció formalmente en 1825 la separación del Brasil.

\section{FACTORES GEOGRÁfićOS}

La política externa de un país está fuertemente influenciada por la geografía. El área de $8.511 .965 \mathrm{Km}^{2}$ del Brasil representa $1,6 \%$ de la superficie total del globo, más del $20 \%$ del continente americano y casi $48 \%$ de América del Sur. Sólo la Unión Soviética, el Canadá, China y los Estados Unidos poseen una extensión territorial superior a la del Brasil. La frontera brasileña es de $15.719 \mathrm{Km}$, siendo la mayor con un solo paf́s, Bolivia, de $3.126 \mathrm{Km}$. y la menor de $593 \mathrm{~km}$., con Suriname. Solamente la Unión Soviética y China tienen mayor número de vecinos fronterizos. Brasil limita con 10 países, es decir, con todos los de Sudamérica, a excepción de Chile y Ecuador. La multiplicidad de vecinos americanos y africanos coloca al Brasil en contacto con una gran variedad de culturas y pueblos.

La forma compacta, casi triangular, de Brasil, contribuyó concomitantemente con la monarquía, à la cohesión interna y a la preservación de la unidad nacional.

El límite marítimo es la mitad del terrestre; los $7.408 \mathrm{Km}$. del litoral se extienden sobre el Atlántico Sur y en pequeña parcela, en el territorio de Amapá, sobre el Atlántico Norte. La costa occidental africana abriga cerca de 20 repúblicas vecinas de ultramar y todavía hay 6 países del Continente Negro vueltos hacia el Atlántico. A través del Océano Atlántico, Brasil se articula directamente con más de 50 países situados en América, Europa y Africa. Entre la ciudad brasileña de Natal y la senegalesa de Dakar, median unos $3.500 \mathrm{Km}$. Durante la Segunda Guerra Mundial, el aeropuerto de Natal fue de extraordinaria importancia estratégica. En Brasil, los extremos Norte y Sur están a una distancia de $4.320 \mathrm{Kms}$. y entre Este y Oeste a $4.328 \mathrm{Kms}$.

Por razones geográficas y otras, América Latina y Africa son prioridades naturales de la polftica externa del Brasil. Comparado con

$$
\left[\begin{array}{lll}
4 & 6 & 0
\end{array}\right]
$$


el Atlántico Norte, el Atlántico Sur es un área de pocas tensiones y Brasilia se empeña en contribuir a la paz en el Attántico Sur y en impedir que se transforme en teatro de bases y confrontamientos militares o en región de guerra fría entre las dos superpotencias o entre países de Occidente.

En este contexto, Itamaraty (Ministerio de las Relaciones Exteriores) se opone a la creación de una organización del Tratado del Atlántico Sur (OTAS), análoga a la OTAN, y rechaza particularmente cualquier vinculación con el régimen racista de Africa del Sur.

\section{FAcTORES ECONÓNirrcos}

Para la caracterización de la política externa del Brasil, son relevantes algunos datos sobre su situación económica, doméstica, incercambio comercial, deudas, reservas monetarias, etc.

En precios constantes, el Producto Interno Bruto pasó de 210.117,9 millones de cruzeiros en 1970, a 480.959,9 millones en $1980^{2}$. El PrB en 1981, teniendo en cuenta el valor real del dólar del año anterior, alcanzó el equivalente a us $\$ 194.171 .000 .000$. Este PIB coloca al Brasil en noveno lingar en el mundo; el PIB "per cápita" fue de 1.574 dólares ${ }^{3}$.

En 1979 el valor de la producción industrial del Brasil correspondió a la 13a del mundo. En el período de 1970 a 1976 la media anual de crecimiento fue de $11,6 \%$. En $197639 \%$ del pis se originó en la industria. Sin embargo, apenas $11,0 \%$ de su fuerza de trabajo se empleó en la industria en este período 4 .

En la industria del acero el país subió del $23^{\circ}$ lugar mundial en 1965 al $13^{\circ}$ en $1979^{\circ}$. La producción nacional de acero creció $62,4 \%$ entre 1974 y 1978 , y en 1979 fue de 13,9 millones de toneladas. En este año Brasil se tornó autosuficiente en productos de acero, pero no en equipos de fábricas siderúrgicas. La previsión para 1984 es de 22 millones de toneladas, igual a la producción del Reino Unido en 1979. A pesar de esto, el consumo per cápita de acero en Brasil es bajo. En 1979 fue de $98 \mathrm{Kg}$. por habitante, "inferior a los niveles de España (305 por habitante) e Italia (398 Kg. por habitante) ${ }^{6}$.

MFigueiredo, João, Mensagem ao Congreso Nacional. Brasilia. Departamento de Imprensa Nacional, 1982, p. 222.

${ }^{3} \mathrm{El}$ proceso de Integración en América Latina. Buenos Aires, INTAL - BID, 1982 , p. 28.

"Selcher, Wayne. "Brazil in the World: A Ranking Analisis" Brazil in the International System: The Rise of a Middle Power. Editado por Wayne Selcher Boulder, Colorado, Westriew Press, 1981, p. 32.

Idem. Ibidem, p. 36.

"Datos citados por Wayne Selcher, "Brazil in the World: A ranking analysis Brazil in the International System: The Rise of a Middle Power. Editado por Wayne Selcher, Boulder, Colorado, Westview Press, 1981. pp. 36-37.

$$
\left[\begin{array}{lll}
4 & 6 & 1
\end{array}\right]
$$


A pesar de su reciente fundación (1957), la industria automotriz brasileña ya se encuentra entre las 10 mayores del mundo. En 1979 fueron fabricados más de un millón de automóvilesit.

El petróleo constituye cerca del $40 \%$ del total de la energía básica consumida en Brasil $^{8}$. El país produce menos del $25 \%$ del petróleo que utiliza. Las importaciones de petróleo pasaron de 769 millones de dólares en 1973 a más de 2.900 millones al año siguiente, aunque el volumen importado fue prácticamente el mismo ${ }^{9}$, a pesar de que todavía es posible substituir el $40 \%$ del petróleo importado por electricidad, carbón mineral y vegetal, combustibles ĺquidos, etc. . 10. El consumo nacional de derivados del petróleo presentó en 1981 una reducción de más del $7 \%$ en relación al año anterior. La represa paraguayo-brasileña de Itaipú, en el río Paraná, está programada para una capacidad de 12 millones y 600.000 Kw. Con precios de enero de 1981, el costo total es de 12 billones de dólares, a pesar de que cerca del $70 \%$ de los gastos son en cruzeiros. En 1983 están programadas para entrar en funcionamiento las dos primeras turbinas.

En orden de importancia, los mayores inversionistas del Brasil son: Estados Unidos (casi 5.000 millones de dólares), Alemania Occidental, Suiza, Japón (cerca de 2.000 millones), Reino Unido y Francia. Toda Europa, con cerca de 7.000 millones de dólares, constituye aproximadamente la mitad de las inversiones extranjeras en Brasil.

Entre 1970 y 1980 lass exportaciones del Brasil crecieron 667\%, saltando de 2.700 a 21.000 millones de dólares. Las importaciones crecieron aún más, generando en la década un déficit de 2.800 mil millones de dólares ${ }^{11}$. Esto se debe, en parte, a la cuadriplicación del precio del petróleo en 1973. De 1979 a 1981, el saldo negativo brasileño solamente con Arabia Saudita, superó los 11.000 millones de dólares ${ }^{12}$.

En 1981 las exportaciones y las importaciones del Brasil fueron, respectivamente, de 23.300 y 22.100 millones de dólares ${ }^{13}$. Así, hubo un importante superávit en la balanza comercial, a pesar de una

Tdem Ibidem, p. 37.

spena, João Camilo. Introduction to Brazil. Grongula Editions I. Estocolmo, Embajada del Brasil, 1982, p. 7.

${ }^{\circ}$ Franco, Alvaro da Costa. "O Brasil e a actual conjuntura económica mundial”. Revista brasileira de Estudos Políticos, No 47: 206, jul. 1978.

20pena, João Camilo. Op. Cit. p. 8.

1Tamer, Alberto. "Brasil Crescimento e Dependência". Estado de São Paulo, $5 / 2 / 81$, p. 145 .

12"Itamaraty divulga programa de Guerreiro no exterior". Jornal de Brasilia, $9 / 10 / 82$, p. 14.

${ }^{13} \mathrm{El}$ proceso de Integración de América Latina, en 1981. Buenos Aires, INTALBID, 1982, p. 28. 
caída del $7,4 \%$ de los precios pertinentes a las exportaciones y a los aumentos del $13,2 \%$ de las importaciones ${ }^{14}$.

En las últimas décadas, Brasil se ha empeñado en diversificàr a sus socios comerciales, y la pauta de sus exportaciones. Individualmente, Estados Unidos continúa siendo el principal importador del Brasil. Sin embargo, el porcentaje de las exportaciones destinadas a ese país cayó de $45 \%$, en 1965 , a menos de $20 \%$ en $1981^{15}$.

En 1960 Latinoamérica, Africa y Asia absorbian menos del $5 \%$ de las exportaciones brasileñas. El comercio con el Tercer Mundo pasó de 120 millones de dólares en 1960 a 1.000 millones en 1973 y a más de 5.000 millones en 1980, Brasil se transformó en el país en desarrollo que destina la mayor proporción de su comercio a otros países en desarrollo16. En 1981, el comercio en el sentido sUR-SUR correspondió al $44 \%$ del total de las transacciones efectuadas por el Brasil. Y las exportaciones hacia Latinoamérica (excluyendo países en desarrollo de lengua inglesa y holandesa del Nuevo Mundo) superaban en valor a las destinadas a Estados Unidos ${ }^{17}$. El siguiente cuadro, con base en datos de la CACEX (Carteira do Comércio Ex-

\begin{tabular}{|c|c|c|}
\hline $\begin{array}{l}1980 \text { (millones } \\
\text { de dólares) }\end{array}$ & $\begin{array}{l}\text { Porcen- } \\
\text { tajes }\end{array}$ & $\begin{array}{l}1981 \text { (millones } \\
\text { de dólares) }\end{array}$ \\
\hline
\end{tabular}

Total de las exportacio.

nes del Brasil

$\begin{array}{llll}20.132 .401 & \text { aprox. } & 23.293 .037 & \text { aprox. } \\ 5.466 .108 & 27,1 \% & 5.936 .311 & 25,5 \%\end{array}$

Europea

Latinoamérica, todos los paises del Nuevo Mundo, menos USA/Canadá

U S A

Asia (exclusire Medio

Oriente)

Europa Oriental

Africa (exclusive Medio

Oriente)

Medio Oriente

Otros

$\begin{array}{rrrr}3.649 .747 & 18,1 \% & 4.403 .853 & 18,9 \% \\ 3.509 .577 & 17,4 \% & 4.404 .233 & 17,4 \% \\ & & & \\ 1.987 .400 & 9,8 \% & 2.261 .745 & 9,7 \% \\ 1.306 .561 & 6,5 \% & 1.698 .771 & 7,7 \% \\ & & & \\ 1.154 .300 & 5,7 \% & 1.695 .357 & 7,7 \% \\ 1.039 .491 & 5,1 \% & 1.249 .757 & 5,3 \% \\ 2.019 .217 & 10,1 \% & 2.007 .010 & 8,8 \%\end{array}$

14Figueiredo, João. Mensagem ao Congreso Nacional. Brasilia DIN, I982, p. 12.

${ }^{16 E n t r e v i s t a ~ d e l ~ E m b a j a d o r ~ P a u l o ~ T a r s o ~ F l e c h a ~ d e ~ L i m a . ~ V i s a ̈ o, ~ x x x ~(50): ~}$ ,30. 14/12/81. A pesar de que el Brasil significa sólo el $1,5 \%$ del total de las importaciones de los Estados Unidos, conforme afirma Leonardo Mota Neto en "Diálogo Brasil y Estados Unidos", Jomal de Brasilia, 26/09/82, p. 8.

${ }^{10}$ Sardenberg, Ronaldo Mota. "A Política Externa do Brasil nas últimas décadas" Revista do Se rviço Público, Brasília, vol. 109.38 (4) ; 29 out. dez. 8I.

${ }^{17}$ Guerreiro, Ramiro Sarziva, "Política Externa do Brasil". Segurança e Desenvolvimento XxxI, (190) 43, 1982. 
terior do Banco do Brasil) ilustra la diversidad de los importadores brasileños:

A pesar de la recesión mundial, inflación generalizada, desequilibrios financieros y proteccionismo, entre 1971 y 1980 el comercio exterior de Brasil creció en $700 \%$, pasando de 6.000 a 43.000 millones de dólares. En este período el comercio del país con los demás países en desarrollo se expandió en $1.700 \%$. Gran parte de este aumento se debió a las importaciones de petróleo, pero también a las exportaciones del Brasil hacia los países en desarrollo, que crecieron en $1.300 \%$ en apenas 10 años, con un salto de más de 6.000 millones de dólares ${ }^{18}$.

Brasil exporta actualmente menos de un décimo del valor de su Producto Interno Bruto, proporción sustancialmente inferior a la de la mayor parte de los países industrializados, teniendo además una pequeña participación en el mércado internacional. Sus exportaciones alcanzan sólo a un $1 \%$ del total de las exportaciones mundiales $^{19}$.

Entre los países en desarrollo, Brasil ocupa el primer lugar como importador y el octavo como exportador (superado apenas por 7 exportadores de petróleo). Después de Estados Unidos, el Brasil es el mayor exportador de productos agrícolas. En el período de 1970 a 1980, las ventas externas brasileñas de manufacturas ascendieron de 614 millones de dólares a 11.300 millones $^{20}$.

El café cayó del $53 \%$ del total de las exportaciones del Brasil, en 1954, al 20,1\% en 1973 y al 10,8\% en $1975^{21}$. En 1981, en el período de enero a octubre, la soya superó al café en importancia en la exportación ${ }^{22}$. En 1981, las proporciones correspondientes al valor de las exportaciones fueron las siguientes: $38,3 \%$ para productos básicos; $8,9 \%$ para semimanufacturados; $51,2 \%$ para manufacturados, y $1,6 \%$ para operaciones especiales ${ }^{23}$.

Los datos siguientes relacionan el monto de la deuda externa brasileña con el PIB, las reservas monetarias y las exportaciones de] país.

El cuadro económico actual del mundo es sombrío. Entre 1945 y 1970, los aumentos de los niveles de los precios eran moderados; las tasas de cambio más estables; la oferta de empleo más amplia. En las décadas de 60 a 70 hubo expansión media de $7 \%$ del comer-

19Idem. Ibidem: pp. 42-43.

${ }^{10}$ Lima, Paulo Tarso Flecha de. "Comércio Exterior do Brasil. Revista Brasileira de Estudos Politicos. (UFirG) No 54. 30/1/1982.

${ }^{30}$ Tamer, Alberto. "Brasil, Crescimento e Dependência". Estado de São Paulo, 5/02/1981, p. 145 .

rSelcher, Wayne. Brazil's Multilateral Relations. Bouder, Westview Press, 1978 , p. 122.

2Para Ia venta de us\$ 1.484 .528 .000 de café hubo us\$2.839.966.000 de soya. Figueiredo, João. Mensagem ao Congresso Nacional, Brasilia, DrN. 1982. p. 233.

Idem, Lbidem, p. 247. 
José Carlos Brandi Aleixo / Lineas generales de la polftica externa del Brasil

cio internacional. En 1980 el aumento fue apenas de 1,5\%. Si en 1978 el crecimiento económico de los países desarrollados de la

DIMENSION DE LA DEUDA24

$\begin{array}{rrc}\text { Año } & \text { Deuda } & \text { Participación en el PIB } \\ 1964 & 3.100 & 24,9 \% \\ 1969 & 4.400 & 14,4 \% \\ 1973 & 12.600 & 15,4 \% \\ : 1981 & 61.400 & 29,7 \%\end{array}$

DEUDAS, EXPORTACIONES Y RESERVAS2

En millones de dólares

$\begin{array}{lccc}\text { Afio } & \text { Deuda } & \text { Reserva } & \text { Exportaciones } \\ 1964 & 3.100 & 244 & 1,4 \\ 1973 & 12.600 & 6.400 & 6,1 \\ 1981 & 61.400 & 7.500 & 23,3 \\ & & & \\ \text { nto en el periodo } & 1.880 \% & 2.970 \% & 1.529 \%\end{array}$

OCDE fue de casi $4 \%$, en 1981 el crecimiento mencionado bajó a 1,2\%. El desempleo en varios de estos países, alcanzó e incluso superó el $10 \%{ }^{26}$.

En los 25 años siguientes al término de la Segunda Guerra Mundial, el sistema financiero internacional particular financió en gran parte la expansión industrial del Tercer Mundo. Muchas empresas, evitando la intensa movilización social de los países más desarrollados, invirtieron muchos recursos en países de mano de obra barata. A partir de 1973, la crisis del petróleo y la desestabilización del sistema monetario fundamentado en el dólar, provocaron grandes desequilibrios. Anteriormente el reciclaje de la deuda externa se operaba como corrección de un desequilibrio transitorio de la balanza comercial. En el caso del Brasil y de varios países del Tercer Mundo, este desequilibrio se tornó estructural. Sólo los intereses de las deudas externas absorben muchas veces del 30 al $60 \%$ del valor de las exportaciones. Se impone, pues, la implantación de un nuevo modelo financiero internacional a través de decisiones políticas apropiadas. Pảgina 15.

sIdem, Ibidem.

${ }^{20}$ Guerreiro, Ramiro Saraiva. Conferência en la Escuela Superior de Guerra. Rio de Janeiro, $3 / 9 / 82$, p. 9 y 10 texto mecanografiado. 


\section{Dimensiones Generales de la Política Externa}

Algunas dimensiones importantes de la política externa del Brasil son consecuencia de su condición asumida de país latinoamericano, atlántico, lusoparlante, pluxirracial, cristiano, occidental y en desarrollo. Tal vez la principal característica del Brasil sea la de la diversidad, mutiplicidad y polivalencia de sus dimensiones. Vale resaltar también la presencia actuante de Brasilia en las Naciones Unidas y en organismos regionales como OEA, BID, SELA, ALADI, Organizaciones del Tratado de la Guenca del Plata, Tratado de Cooperación Amazónica. En la OEA, el Presidente Juscelino Kubistschek inició, en 1958, la Operación Panamericana (OPA). De ella surgió el Comité de los 21, encargado de estimular la cooperación económica entre los países miembros. El Brasil dio un gran apoyo a la creación de la Comisión de la onu sobre Comercio y Desarrollo (UNCTAD) ${ }^{27}$.

En los discursos presidenciales y de los cancilleres brasileños, así como en declaraciones o comunicados conjuntos con autoridades de otros países, hay frecuentes menciones de los principios y normas de la política externa del Itamaraty. Muchos de ellos se encuentran en documentos internacionales tales como las Cartas de la onU y de la OEA, por ejemplo: autodeterminación de los pueblos, no intervención, condenación del uso de la fuerza o de la amenaza de su empleo en las relaciones internacionales, solución pacífica de las controversias, fiel cumplimiento de los tratados, implantación de buena fe de las obligaciones internacionales libremente asumidas, respeto a la soberanía de los países, seguridad colectiva, incluso eco. nómica; igualdad de las naciones, rechazo de la hegemonía propia o ajena, así como de cualquier forma de interdependencia vertical.

La Cancillería brasileña procura evitar los extremos de las fórmulas generales y acabadas, y de las causalidades unilaterales. Además, afirma la compatibilidad de los intereses de las naciones. Dentro de los principios generales se buscan acuerdos concretos, viables y mutuamente beneficiosos para las partes. Se aboga el respeto pleno a la individualidad de las partes y la comprensión de sus realidades específicas. Se niega en el análisis de problemas ajenos cualquier pretensión de juez o maestro.

Se rechaza la visión de la política internacional como un juego de poder, no se pregonan formas abstractas de equilibrio, constitución de bloques, confrontaciones o reduccionismos ideológicos. No se acepta una correlación necesaria entre mayor poder y mayor moderación. Se atribuyen las amenazas a la paz internacional, particularmente a la carrera armamentista, a la persistencia de las desigual-

"Trindade, Antônio Augusto Cançado. "Posições Internacionais do Brasil no plano Multilateral. Revista Brasileira de Estudos Politicos. 52: 147-219 Enero 1981. 
dades económicas, al enraizamiento de las crisis regionales'y a la fragilidad de los mecanismos encargados de superar las dificultades $^{28}$.

La condena del congelamiento del poder, la búsqueda de un nuevo orden internacional y un moderado optimismo al respecto, aparecen en los pronunciamientos de cancilleres brasileños. Ejemplo de lo anterior, es el siguiente trozo del discurso del actual. Canciller Saraiva Guerreiro, el 30 de noviembre de 1981:

"El Brasil cree que las estructuras del sistema internacional pueden ser transformadas, a pesar de ser relativamente rígidas... En realidad, dos procesos corren paralelos. De un lado la politización del inmovilismo, o sea, la resistencia a los procesos de cambio, resistencia adoptada políticamente, por un acto de deliberación política. De otro, las centativas de cambio.

... El sistema internacional está estructurado en términos de jerarquía de poder... esas jerarquías entre las naciones traducen a corto plazo ventajas de tipo económico y político. Por eso la superación de Ios clesacuerdos del sistema internacional no es entendidá como una nueva forma de organización del sistema internacional que puede beneficiar a todos, sino que simplemente como una amenaza a las posiciones del poder efectivo, que se verían muy debilitadas por las transformaciones"'29.

En una conferencia pronunciada este año en la Escuela Superior de Guerra, el Canciller Saraiva Guerrero afirmó:

"En la perspectiva brasileña, el actual estado crítico de la economía internacional, aunque particularmente nefasto para las economías en desarrollo, es indeseable para la comunidad internacional en su conjunto. Es a partir de esta visión integrada, y que nos parece racional y realista, que el Brasil reafirmó la convicción de que la cooperación internacional no es sólo el corolario de abstractos ideales humanitarios de solidaridad, sino la inevitable consecuencia de un diagnóstico global de la realidad económica internacional"30.

No se niega la importancia de la recuperación de las economías deI Norte para revertir el cuadro actual de estancamiento. Se exige, empero, un tratamiento global e integrado de los principales temas de la realidad internacional, inclusive de los problemas económicos que, aunque con repercusiones de gravedad distinta, afectan a los países del Norte y del Sur. En la óptica brasileña, el diálogo comporta una dimensión ideal, que se traduce en la búsque-

\footnotetext{
${ }^{29}$ Guerreiro, Ramizo Saraiva. Discurso en la Escuela Superior de Guerra, Rio de Janeiro, 3/9/82 p. 19 texto mecanografiado.

${ }^{20}$ Guerreiro, Ramiro Saraiva, Conferencia del Canciller en Londres en el Real Instiluto de Relaciones Internacionales, (Chattam House) 20/11/81. Texto mimeografiado. Pp. 5 y 7.

$303 / 9 / 82$, texto mecanografiado, P. 9.
} 
da de la equidad entre las naciones, pero comporta, igualmente, una vertiente práctica, reflejada en el esfuerzo para obtener beneficios comerciales visibles y concretos.

\section{la Dimensión Occidental}

No existe un amplio acuerdo sobre el significado de Occidente. Consideraciones de carácter etimológico, geográfico, Iingüístico, religioso, económico, militar, político, cultural y filosófico pueden ayidar a comprender el complejo concepto de Occidente. Sobre ello escribieron autores diversos, tales como: Roger Bastide ${ }^{31}$, Roger Garaudy ${ }^{32}$, James Fulbright ${ }^{33}$, Louis Joseph Lebret ${ }^{34}$, Julián Marías $^{35}$, Oswald Splenger ${ }^{36}$ y Arnold Toynbee ${ }^{37}$. En Brasil; entre otros, estudiaron el tema Golbery do Couto e Silva ${ }^{38}$ y J. O. de Meira Penna ${ }^{39}$.

Puesto que el concepto de Occidente se ha desvinculado de su connotación geográfica inicial, se entiende por qué el nazismo se opuso simultáneamente a él y a la ideología marxista-leninista del Este Europeo ${ }^{40}$.

La condición de país occidental es afirmada reiteradas veces por líderes y gobernantes brasileños. En razón de la natural brevedad de espacio, este trabajo se limita a algunos pronunciamientos más recientes sobre el tema.

Ya en 1958, en clima de guerra fría, en una conferencia sobre "El Brasil y la defensa del Occidente", escribió Golbery do Couto e Silva: "El único Occidente que vale como un todo duradèro y cohesionado, el Occidente que se puede de hecho distinguir nítidamente de tantas otras civilizaciones y culturas, dotado de una individualidad propia, original y marcadamente característica, es para nosotros el Occidente como ideal, el Occidente como propósito, el

${ }^{1}$ Le Prochain et le Lointan. Paris Edition, Cujas, 1970.

${ }^{32} O$ Ociclente é um Acidente. Rio de Janeiro, Salamandra, 1978.

${ }^{39}$ As perspectivas do Ocidente. Rio de Janeiro, Ed. Record, 1964.

${ }^{3}$ Suicidio ou Sobrevivência do Ocidente, Problemas Fundamentais de nossa civilização. São Paulo, Duas Cidades, 1964.

* O Ocidente e seus Inimigos. Visão, 14/9/81. pp. 70-72.

${ }^{8} A$ Decadência do Ocidente, Rio de Janeiro, Zahar, 1978.

${ }^{37}$ Estudos de História Contemporânea. $A$ Givilização a prova. O Mundo $e$ ocidente. São Paulo, Companhia ed. Nacional, 1976.

ss" O Brasil e a Defesa do Ocidente" Geopolitica do Brasil. Rio de Janeiro, José Olympio, 1967, p. 225.

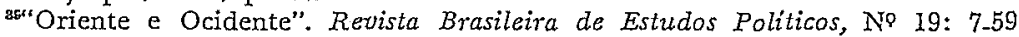
jun. 1965.

${ }^{10}$ Dijo el Führer a un extranjero el 9 de agosto de 1939: "todo lo que emprendo es dirigido contra Rusia. Si el Occidente es necio y demasiado ciego para entender eso, estaré obligado a entenderme con Rusia, vencer al Occidente, después reunir mis fuerzas y volcarme contra la Unión Soviética". Fest, Joaquim. Hitler. Rio, Nova Fronteira, 1976, p. 696. 
Occidente como programa... Resumiéndolo en sus términos esenciales: la ciencia como instrumento de acción; la democracia como fórmula de organización política; el cristianismo como supremo padrón ético de convivencia social. Y ahí están contenidas, en sus justos límites, la libertad, igualdad y fraternidad; el amplio reconocimiento de la dignidad del hombre; la plena expansión de la personalidad individual; el máximo de bienestar físico y espiritual para todos; justicia social y paz"41.

Aún en el período de particular aproximación con los Estados Unidos, el Presidente Castello Branco distinguió entre intereses comunes del Occidente y los específicos de una Gran Potencia: "No debemos comprometer nuestra actitud ni por maquiavelismo provinciano, ni por una política de extorsión. Recíprocamente, no debemos adherir previamente a las actitudes de cualquiera de las grandes potencias, ni siquiera a las potencias guardianas del mundo occidental, pues en la política externa de éstas es necesario distinguir los intereses básicos del sistema occidental de los intereses específicos de una gran potencia"42.

A continuación, reproducimos las palabras del ex Canciller Azeredo da Silveira en presencia de las Comisiones de Relaciones Exteriores del Senaclo y de la Cámara:

"Los propios ideales que son la médula de este concepto filosófico y humanista que es el del Occidente, excluyen la idea de monolitismo rígido. El respeto a la diversidad de situaciones y de opciones es una de las características fundamentales de la concepción de la vida que, a lo largo de los siglos, se fue destilando en estas vastas áreas del planeta que forman hoy el mundo occidental.

"A mi entencler, el propio concepto de Occidente no es un concepto éstático, geográfica o históricamente hablando. Descle el punto de vista geográfico, se amplió considerablemente, e incluye hoy naciones que se sitúan fuera de los meridianos que lo limitaban, inicialmente, a Europa y, después, a ésta y al continente americano. Históricamente el Occidente se embebe de experiencias que le traen naciones nuevas. Tales experiencias, diferentes de las que ocurrieron en su propio territorio inicial, contribuyen al enriquecimiento y vitalidad del mundo occidental. Los padrones de convivencia racial desarrollados en el Nuevo Mundo, sobre todo en nuestro país, representan un factor nuevo que ilustra esa contribución" 48 .

Los países del Primer Mundo no serán fieles a los propios ideales del Occidente si toleran cualquier forma de colonialismo y si

${ }^{4 G e o p o l i t i c a ~ d o ~ B r a s i l . ~ R i o ~ d e ~ J a n e i r o, ~ J o s e ́ ~ O l y m p i o, ~ 1967, ~ p p . ~ 225-226 . ~}$

*Diskurso de 31/7/1964. Discursos de 1964, Brasillia, Secretaria de Imprensa, s.d., p. 110 .

anesenha de Polltica Exterior do Brasil. Brasilia, MRE No 10: 60, julio, agosto, setembro. 1976 . 
no consienten en los cambios estructurales de orden internacional. Caben aquí dos citaciones elocuentes del Canciller Saraiva Guerreiro:

"En una evaluación de más largo alcance, el más expresivo resultado de Cancún tal vez haya sido alertar a los países del Norte $y$; especialmente, al gobierno norteamericano, sobre el hecho de que la idea abstracta de supervivencia de los valores del Occidente debe necesariamente pasar por la materia concreta de transformación de algunas estructuras obsoletas e injustas de la economía internacional. Tal transformación debe ser el modelo para beneficiar a los países del Tercer Mundo, para consolidar y perfeccionar los modelos de cooperación entre el Occidente desarrollado y los países en desarrollo. Es fundamental reforzar los vínculos de confianza entre el Occidente y el Tercer Mundo lo que, dadas las realidades del mundo contemporáneo, requieren mayor flexibilidad en los procesos de negociaciones económicas y políticas con el mundo en desarrollo" 44 .

En una entrevista en noviembre de 1981, el mismo Canciller Saraiva Guerreiro declaró:

"Nada peor para el Occidente que caracterizarse en todas partes como un defensor de ciertas situaciones coloniales, o de hegemonías... No puede haber nada peor a largo plazo. Las posibles ganancias momentáneas son más que perjudicadas por los efectos negativos, por los resentimientos, por la pérdida de la confianza. Es entregar al Este todas las causas bonitas, incluso los propios principios occidentales que son incompatibles con el colonialismo, con la discriminación racial... Son los principios comunes a los países occidentales... libertad, igualdad, cooperación, con vistas al bienestar en una base que no anule al individuo $y$, de cierta forma, la capacidad de iniciativa. En el campo internacional esto debe llevar a la tolerancia con países que, como resultado de su lucha contra el colonialismo, se encaminaron hacia soluciones internas diferentes. Lo importante es que todos ellos acaben adquiriendo confianza en el Occidente, viendo que su seguridad y su posibilidad de evolución no son puestas en riesgo por el Occidente, sino que el Occidente es el que mejor puede cooperar con ellos, respetándoles la personalidad; y es el que más puede ofrecerles en términos de desarrollo económico, de mercados, de cooperación técnica, y así sucesivamente" 45 .

En una conferencia en 1982, a propósito de la visita del Presidente Figueiredo a los Estados'Unidos, afirmó el Canciller Saraiva Guerreiro:

\footnotetext{
H"Os controvertidos resultados de Cancún". Folha de São Paulo, 15/11/81. Página 3.

"Guerreiro diz que Ocidente erra ao apoiar o Apartheid". Jornal do. Brasil, $13 / 9 / 81$, p. 18 .
} 
"En Washington, el Presidente Figueiredo recordó la participación del Brasil en la histórica tarea de construcción del Occidente, mencionando al mismo tiempo que nuestro país comparte los problemas, objetivos y aspiraciones del mundo en desarrollo... El Presidente no dejó de señalar la necesidad de que el Occidente, en el cual los Estados Unidos desempeñan un papel central, trabaje con objetividad y consistencia para restaurar con el mundo en desarro. llo vínculos de mutua confianza"46.

Recientemente afirmó el actual Presidente del Brasil en la onu:

"Integrado en el mundo Occidental, el Brasil desea realizar sus aspiraciones nacionales con pleno respeto a la libertad, a la democracia y a los derechos de la persona humana. Esos altos valores, así como la tradición occidental de pluralismo y de igualdad entre las naciones, forman un cuadro que faculta al Brasil actual fuera del hegemonismo de superpotencias o presiones ideológicas adver$\operatorname{sas}^{\prime \prime 4}$.

Estos y otros discursos demuestran el rechazo brasileño a dos formas existentes de reduccionismo del concepto de Occidente. Dejando de lado ideales universalistas anteriores, algunos quieren equiparar el Occidente a la civilización industrial en las sociedades democráticas y representativas (Europa Occidental, Estados Unidos, Canadá y hasta el Japón). Se llega a pensar que en razón de la escasez de recursos naturales en el mundo, del excesivo crecimiento demográfico en el Sur y de las limitaciones internas numerosas, sólo un grupo reducido de países puede componer el Occidente, al menos a corto y mediano plazo. El otro reduccionismo es de los que equiparan Occidente y antisovietismo, tratando de vaciar de su contenido propio un concepto riquísimo en valores y limitarlo a la negación de una determinada ideologia.

\section{La Dimensión Latinoamericana}

Muchos vínculos aproximan al Brasil de América Latina. Son ejemplos de ello: la proximidad geográfica, el pasado colonial, los idiomas semejantes, el predominio del catolicismo, la condición común de países en desarrollo, la necesidad de mayores mercados y de mayor influencia en los asuntos internacionales, etc.

En el siglo xvir tuvo gran importancia para los países iberoamericanos el Tratado de Madrid, de 1750. El internacionalista Rodrigo Atávio lo considera la Carta Magna de América Latina, por su espíritu de justicia y conciliación. Gupo al brasileño Alexandre de Gusmão, miembro eminente de la delegación Iusitana, un gran mérito por su elaboración. El sintió la singularidad de América, la

tuscuela Superior de Guerra, Rio de Janeiro, $3 / 9 / 82$, p. 18 .

"Estado de Säo Paulo, 28/9/82, p. 6.

$$
\left[\begin{array}{lll}
4 & 7 & 1 \cdot
\end{array}\right]
$$


necesidad de separar los intereses del Brasil de las intrigas dinásticas europeas y la urgencia de resolver irritantes cuestiones de fronteras. En ese contexto merece destacarse especialmente el artículo $\mathrm{xxr}$, en el cual se lee: "siendo la guerra ocasión principal de los abusos y motivos de la alteración de reglas bien concertadas, quieren Sus Majestades Fidelísima y Católica que (que Dios no lo permita) si se llegase a romper entre las dos coronas, se mantengan en paz los vasallos de ambos establecidos en toda la América Meridional, viviendo unos y otros como si no hubiera tal guerra entre los soberanos, sin hacerse la menor hostilidad... la dicha continuación de paz perpetua y buena vercindad no tendrá lugar solamente en tierras e islas de América Meridional". La expresión América Meridional en la época y aún en la primera mitad del siglo xIx era usada para designar los territorios que iban de México a la Patagonia. En verdad sólo en la década de 1850 surge el nombre de América Latina, de la pluma del colombiano José María Torres Gaicedo ${ }^{48}$.

Silvestre Pinheiro Ferreira, el último ministro de asuntos extranjeros nombrado en el Brasil por Don João vr, llegó a designar el agente Schmidt para servir junto a Simón Bolívar. En el año de 1822 sometió a las jóvenes naciones del Continente un proyecto de "Tratado de Confederación y mutua garantía de independencia".

Simón Bolivar, durante algún tiempo, manifestó cierta hostilidad en relación al Brasil, en parte debido al hecho de que don Pedro I era sobrino de Fernando vII y posiblemente vinculado a los absolutistas de la Santa Alianza.

EI rechazo taxativo por parte de Don Pedro I a la propuesta de un comandante español, en 1825, de anexar al Brasil el territorio boliviano de Chiquitos, el conocimiento del texto liberal de la Constitución brasileña de 1824 y la eliminación de equivocaciones contribuyeron a que Bolívar, en sus últimos años, manifestase un gran aprecio por el Brasil. Para esta mayor aproximación fue importante la actuación del pernambucano José Inácio de Abreu e Lima; que defendió al Libertador con la espada y la pluma ${ }^{49}$. A pesar de haber aceptado la invitación del Presidente en ejercicio Francisco de Paula Santander y haber designado como su representante a Teodoro José Biancardí, el Brasil no compareció al Congreso Anfictiónico de Panamá de 1826. Adquirió, sin embargo, y conservó el único texto original no desaparecido de las Actas de la Reunión. Estas están donadas al Gobierno de Panamá y serán guardadas en un monumento de este país dedicado a la memoria del histórico cónclave.

\footnotetext{
${ }^{\star A}$ Ardao, Arturo. Génesis de la Idea y' el Nombre de América Latina. Cara. cas. Centro de Estudios Latinoamericanos Rómulo Gallegos. 1980. p. 73.

${ }^{40}$ Lima, Nestor dos Santos. La imagen del Brasil en las Cartas de Bolivar. Rio de Janeiro. Banco do Brasil s/d.
} 
Las fronteras del Brasil con sus vecinos fueron definidas en tratados bilaterales y demarcadas por comisiones mixtas. No hay problemas de límites.

La suscripción el 19 de octubre de 1979 del Acuerdo de Cooperación Técnico-Operativo para los aprovechamientos hidroeléctricos de Itaipú y Corpus, constituye marco importante en las relaciones del Brasil con Argentina y Paraguay. Fue el resultado de largas negociaciones y demostró la posibilidad de fórmulas mutuamente satisfactorias para litigios muy complejos.

Itamaraty desea la solución pacifica de los puntos de fricción todavía pendientes en América Latina. Acompañó con preocupación el conflicto armado entre Argentina y Reina Unido. Én este caso renovó su reconocimiento, ocurrido ya en 1833, de la soberanía de Argentina sobre las Malvinas.

El Brasil considera importante que las superpotencias no extiendan hacia América Latina, y particularmente hacia Atnérica Central, sus confrontaciones ideológicas y militares. Las tensiones regionales disminuirían mucho si Washington y Moscú no intentasen colocar en la carrera armamentista su seguridad y sí en el diálogo. A pesar de los eslabones arriba mencionados, las economías de los países latinoamericanos fueron orientados no en el sentido de la complementación mutua sino que hacia centros dinámicos extrarregionales y, particularmente, al Reino Unido y después a Estados Unidos.

Sin embargo, en las últimas décadas ha crecido el diálogo de los países Iatinoamericanos entre sí. Brasil procura construir su presencia en el continente sobre la base de la aceptación de la diversidad, de la adaptación a los cambios, de la búsqueda de lo nuevo, del mutuo reconocimiento en todas las dimensiones y, sobre todo, de la identidad común latinoamericana. El intercambio comercial, cultural, político y social ha aumentado en la región. En 1964 el Presidente De Gaulle respondió a su colega Eduardo Frei que mucho le había impresionado cuán poco se reunían los gobernantes de América Latina, en contraste con los numerosos encuentros de las autoridades europeas. La situación varió bastante en los últimos lustros. Los primeros encuentros del Presidente del Brasil con sus colegas de Colombia y Venezuela ocurrieron, respectivamente, en 1971 y 1972. Antiguamente los presidentes brasileños electos o que habían tomado posesión visitaban en primer lugar Europa o los Estados Unidos. El actual mandatario, no obstante, inició su programa de viajes al exterior con una visita a Venezuela. El actual presidente del Ecuador, Dr. Osvaldo Hurtado, en su primera ausencia del país se dirigió al Brasil. El Jefe de Estado del Brasil ya visitó en los tres primeros años de su mandato a varios colegas de América del Sur y México. 


\section{La Dimensión Tercer-Mundista. El Diálogo Norte-Sur}

El mundo puede ser dividido conforme a criterios muy variados. En 1956 el demógrafo francés Alfred Sauvy publicó su trabajo Tiers Monde: sous développement et développement. Se pensó en la analogía entre el Tercer Estado, que era el último en el plan económico, político y social entre los tres Estados generales franceses antes de 1789, y el grupo de naciones cuyo núcleo inicial se reunió en Indonesia, en Bandung, en 1955. El Tercer Estado era el más numeroso y el menos poderoso en el ancièn régime. El Tercer Mundo es el más numeroso y el menos poderoso de la humanidad. A grosso modo constituyen hoy el Primer Mundo los países industrializados del Este Europeo50. El Brasil no ve ninguna incompatibilidad en pertenecer simultáneamente al Occidente y al Tercer Mundo. Pertenece al Occidente por libre opción, por aceptar sus valores filosóficos y humanistas; integra el Tercer Mundo por su condición de país en desarrollo, donde hay lagunas significativas en los campos de alimentación, vivienda, salud, ahorro, alfabetización, tecnología, distribución de renta entre las personas y entre las regiones, etc.

Hay, naturalmente, diferencias entre los países del Tercer Mundo, por ejemplo, el grupo de países exportadores de petróleo (OPEP); el grupo de países recientemente industrializados (NIC's) ; el grupo de países de menor desarrollo (LDC's). Algunos sectores del Primer Mundo enfatizan estas diferencias en el intento de negociar por separado y más ventajosamente con los diversos grupos. En este contexto el Brasil ha pregonado la necesidad de cohesión de todos los componentes del Tercer Mundo.

Son oportunas las palabras del Embajador Flecha de Lima: "El Brasil no se dejará engañar por rótulos de país recientemente industrializado o de país en desarrollo avanzado, rótulos cuyo objetivo es simplemente el de separarnos de los demás países del Sur, y así, con aparente legitimidad, negarnos los beneficios de la cooperación internacional" 51 .

En la onu, el Presidente Figueiredo resaltó la importancia de la cohesión del Tercer Mundo: "En cuanto al Sur, ha llegado el momento de dar expresión efectiva a la cohesión que hemos sabido preservar por encima de las dificultades externas y las diferencias internas. Es necesario caminar hacia la implantación de una interdependencia verdaderamente solidaria entre América Latina, Africa y Asia. Debemos dejar claro con nuestras acciones y con nuestras palabras que la diversidad del Tercer Mundo encierra insospe-

${ }^{\circ}$ Según discursos de China, hay una triple división: Primer Mundo (Dos superpotencias); Segundo Mundo (Países Desarrollados); Tercer Mundo (Demás Países, inclusive China).

"Lima, Paulo Flecha de. "Comercio Exterior do Brasil". Revista Brasileira de Estudos Políticos, No 54: 22-23, enero 1982. 
chadas: oportunidades de complementación económica, y es factor de unión y no materia de maniobras para iniciativas tales como la discriminación arbitraria entre países en desarrollo, que tienden a deshacer al mismo tiempo la cohesión del Tercer Mundo, la cooperación internacional para el desarrollo y el diálogo Norte-Sur" ${ }^{25}$.

El Sur no puede esperar una anterior recuperación del Norte. La recuperación debe ser de todos. Dijo al respecto el Ganciller Saraiva Guerreiro: "Si estamos plenamente conscientes de la importancia de la recuperación de las economías de los países industrializados para el surgimiento de la economía internacional, no concordamos con la idea de que ese fenómeno aislado -la recuperación de las economfas centrales - sea la única salida para la situación de crisis en que nos encontramos, ni que los países en clesarrollo deban esperar pacientemente hasta que los beneficios de la recuperación de los países desarrollados eventualmente lleguen a alcanzarlos. No concordamos tampoco con la tesis de que las fuerzas del mercado, por sí solas, serán capaces de promover la recuperación económica, primeramente a nivel nacional $y$, luego, en consecuencia, a nivel universal"'53.

$\mathrm{Y}$ en el mismo discurso pronunciado en la onv afirmó el Presidente João Figueiredo: "Ios intereses del Norte y del Sur no son contradictorios. En verdad, no hay una crisis entre el Norte y el Sur, sino una crisis profunda que afecta a ambos, una situación terriblemente adversa y de la cual sólo saldrán por el camino del entendimiento y de la solidaridad, nunca por el retorno a la aspereza y a la confrontación" $" 54$.

\section{La Dimensión Africana}

La presencia africana es importantísima en la composición étnica, en la cultura y en la historia del Brasil. 'También merece destacarse, aunque en proporciones mucho menores, la presencia del Brásil en el Continente Negro. En Africa Occidental, particularmente en Nigeria, en Benin, en Togo y en Ghana, existen comunidades descendientes de negros que, después de años de residencia en el Brasil, cruzaron el Océano Atlántico. Valga el ejemplo de Silvanus Olimpio, prócer de la independencia de Togo y su primer Presidente, de origen brasileño ${ }^{55}$.

En todo caso cabe resaltar una diferencia en la evolución histórica del Nuevo Mundo, Brasil inclusive y de Africa. En el siglo xIx, con la salida de las autoridades europeas, el poder político pa-

"Estado de São Paulo, 28/9/82, p. 6.

Saraiva Guerreiro, Ramiro, Esc. 9/9/82, pp. 12.13.

${ }^{\sharp}$ Estado de São Paulo, 28/9/82, p. 6 .

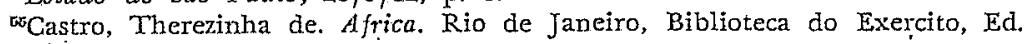
1981. 
só a sus descendientes. En el siglo $\mathrm{xx}$, con la retirada de los europeos, el poder político pasó a los descendientes de los africanos que allí vivían en el inicio de la colonización.

Hasta la década de 1950, gran parte de las relaciones del Brasil con Africa se hizo a través de las metrópolis europeas. Después de la Segunda Guerra Mundial crecieron en Africa y en Asia los movimientos nacionalistas. La Asamblea General de la ONu aprobó, mediante Resolución 1.514 (xv), de 14 de diciembre de 1960, la "Declaración que otorga la independencia a los países y a los pueblos coloniales". En ella "se proclama la necesidad de poner fin incondicionalmente y con rapidez al colonialismo, bajo todas sus formas y en todas sus manifestaciones". La Resolución contó con el apoyo definido del Brasil. Al año siguiente, el nuevo Presidente Jânio Quadros se empeñó en multiplicar los vínculos con Africa y dio a conocer a Lisboa la simpatía del Brasil por la emancipación de los territorios portugueses de ultramar. En 1972, el Canciller Mario Gibson Barbosa visitó Costa de Marfil, Senegal, Ghana, Togo, Benin, Zaire, Gabón, Camerún y Nigeria. Sus sucesores, Azeredo da Silveira y Saraiva Guerreiro, ya cruzaron el Atlántico en viajes oficiales a diversos vecinos de ultramar. Ministros de otros portafolios y frecuentes misiones comerciales incluyeron Africa en sus programas de contactos con el exterior. Brasilia, sobre todó en los últimos diez años, acogió a varios Jefes de Estado y de Gobierno, así como a numerosos ministros de Africa. En 1979, Brasil recibió un total de 18 delegaciones sólo de Nigeria, su mayor "socio" comercial en el continente negro.

En los últimos años fueron creadas varias líneas de navegación y dos líneas aéreas para Lagos y Luanda, esta última ya en 1981.

A principios de 1982, Brasil mantenía 19 Embajadas residentes en Africa y programaba elevar este número a 21, con la inclusión de Zambia y de la República del 'Camerún. Además, las 19 Embajadas representaban al Brasil en otros 22 países africanos donde todavía no había misiones residentes.

La política del Brasil con relación a Africa se inspira en los principios generales de la diplomacia mencionados anteriormente. Brasil y los países africanos condenan hegemonías, paternalismos $y$ cualquier forma de neocolonialismo, y se niegan a ser instrumentos de las superpotencias. Brasil procura estar presente en Africa en la medida en que sus países así lo deseen. Se defiende un Atlántico Sur abierto al intercambio pacífico en todos los campos.

A pesar de la condición común de países en desarrollo, hay cierta complementación en las economías. Brasil puede importar metales no ferrosos, petróleo, fosfato, ciertas maderas tropicales, etc, y el continente vecino puede recibir manufacturas, diversos servicios, algunos alimentos, etc. Hay también campo para operaciones triangulares. Un ejemplo es el proyecto brasileño de prestación de 
servicios en el sector de carbón de Mozambique, financiado con fondos de la OPEP.

En este contexto declaró el Canciller Saraiva Guerreiro:

"El Brasil suministra servicios de Ingeniería y Gonsultoría fácilmente adaptables a Africa. La cooperación también se desenvuelve en la formación de técnicos, en el entrenamiento de nivel medio, el planteamiento de sistemas de enseñanza, etc. Las empresas brasileñas en Africa se adaptan con gran facilidad y adquieren experiencias importantes para el Brasil. Tales contactos son positivos para los dos lados.

El total del intercambio pasó, de cerca de 700 millones de dólares en 1979, a más de 1.400 millones en 1980, o sea, se duplicó en un año"s6.

Las exportaciones del Brasil para el continente africano aumentaron en el período de enero a septiembre de 1981 en más de $50 \%$ en comparación con el año anterior ${ }^{57}$.

En el discurso del 27 de septiembre de 1982, en la Asamblea General de la oNu, el Presidente João Figueiredo renovó las críticas del Brasil a la actuación de Africa del Sur, aunque sin citar expresamente nombres:

"También en Africa Austral persisten focos de tensión generados por la ocupación de Namibia y por sucesivos actos de agresión a países independientes, principalmente Angola. El Brasil no puede dejar de condenar igualmente la discriminación racial, en particular sus formas institucionalizadas, que amenazan la propia paz internacional" 68 .

\section{La Dimensión Lusopariante}

Los portugueses llevaron su lengua a todos los continentes del mundo. En 1822, con la independencia del Brasil, surgía el segundo Es. tado lusoparlante. Lisboa y Río de Janeiro, en los siglos $\mathrm{xIx}$ y $\mathrm{xx}$, firmaron numerosos acuerdos comerciales, económicos, sociales y culturales. En los años 1974 y 1975 surgieron en Africa cinco paises de lengua portuguesa: Guinea Bissau (10/9/74), Cabo Verde $(6 / 6 / 75)$, Santo Tomás y Príncipe $(12 / 7 / 75)$, y Angola (11/11/75). Brasilia rápidamente reconoció su independencia y estableció misiones diplomáticas ante los nuevos gobiernos.

El uso del mismo idioma facilita la cooperación mutua. Representantes oficiales de los siete países de lengua común ya se reunie-

${ }^{56}$ Saraiva Guerreiro, Ramiro. Entrevista del Canciller. Folha de Säo Paulo, $13 / 9 / 81$, p. 3 (Folhetim).

"Saraiva Guerreiro, Ramiro. "Diálogo de Confianza y Amistad". Afrochamber. Enero/Febrero, I982, p. 5.

${ }^{s}$ Estado de Säo Paulo, 28/9/82, p. 6 . 
ron en Portugal y en Cabo Verde para la elaboración de un texto único en portugués sobre el derecho del mar. La IIr Conferencia de los Jefes de Estado de las cinco Repúblicas africanas arriba mencionadas, realizada en la ciudad de Praia en los días 21 y 22 de septiembre de 1982, abordó la conveniencia de introducir el portugués en las organizaciones internacionales como lengua de trabajo. Además de decidir emprender acciones con miras a materializar este objetivo, se subrayó la importancia de la creación de mecanismos de consulta a los otros países de lengua portuguesa. Ya está en preparación un Encuentro de Ministros de Justicia de estos países.

Brasil mantiene contactos con los países de lengua portuguesa en Nueva York, en la sede de las Naciones Unidas. Las relaciones bilaterales crecieron y hay programas de cooperación técnica y cultural con los países africanos de expresión común. El Canciller Saraiva Guerreiro ya visitó Luanda y Maputo, y los Cancilleres de Mozambique y Angola fueron recibidos en el Brasil. Anteriormente, el Presidente de Guinea Bissau estuvo en Brasilia y en otras ciudades del país.

\section{Otras Dimensiones}

Aunque brevemente, cabe aquí añadir algunas consideraciones sobre otras de las numerosas dimensiones de la política externa brasileña.

La población de origen árabe es muy expresiva. La influencia árabe llegó al Brasil a través de Portugal, donde vivieron los mahometanos durante varios siglos, y ella está presente en el vocabulario, en las costumbres y en la cultura en general. Terminada la Segunda Guerra Mundial, el 29 de noviembre de 1947 el Brasil votó a favor de la Resolución $N^{\circ}$ 181, que prevéra la partición de Palestina en dos Estados (uno árabe y otro judío) y la creación de un Corpus Separatrum para Jerusalén y algunas áreas circunvecinas. Itamaraty apoyó la Resolución 242, de 22 de noviembre de 1967 del Consejo de Seguridad de la onv, que enfatiza la inadmisibilidad de la adquisición de territorios por la guerra ${ }^{59}$ y exige la retirada de Israel de los territorios ocupados en reciente conflicto. Brasil reconoce la Organización para la Liberación de Palestina como la legítima representante del pueblo palestino.

Desde principios del siglo $\mathrm{xx}$ Brasil acogió millares de inmigrantes japoneses. Sus descendientes superaron el millón, y ocupan Iugar importante en la economía del país. El comercio con el Japón es muy grande, sobre todo teniendo en cuenta la distancia geográfica. Hay también mucho capital japonés en el país.

${ }^{\varpi} \mathrm{E}$ l parágrafo único del Artículo 79 de la Constitución del Brasil dice: "Está prohibida la guerra de conquista". 
En 1974, fueron reanudadas las relaciones diplomáticas entre Brasilia y Pekín, en clima de cordialidad. Desde entonces ha habido numerosas visitas de autoridades brasileñas a China, y viceversa. Existe un enorme potencial para mayor intercambio comercial $y^{\prime}$ científico.

Históricamente, las relaciones entre Brasil y los países del Este europeo han sido limitadas. Después de la revolución de 1917, Brasil retiró a su Embajador de Rusia. El nuevo régimen soviético sólo fue reconocido al final de la Segunda Guerra Mundial, pero en 1947 cesaron nuevamente las relaciones diplomáticas, que fueron reanudadas en 1961. En los últimos años, varias misiones brasileñas han visitado el Este europeo. En octubre de 1982, el Vicepresidente de la República visitó Hungría, y el Presidente de Rumania vino al Brasil. El saldo comercial ha sido favorable al Brasil. En mensaje al Gongreso Nacional escribió el Presidente Figueiredo: "Las relaciones del Brasil con los países socialistas de Europa Oriental fueron marcadas en 1981 por la expansión y diversificación. Se multiplicaron las visitas oficiales y se incrementó el intercambio comercial que, llegando a cerca de us $\$ 1.600$ millones, alcanzó los niveles más elevados de las últimas décadas" ${ }^{\prime 0}$.

\section{Consideraciones finales}

En las páginas anteriores se enfatizaron algunas de las características más constantes de la política externa brasileña. Tal vez la principal de ellas sea su vocación universalista, basada también en la multiplicidad de grupos étnicos presentes en su historia y hoy integrados por una única lengua y nacionalidad. Contando probablemente con la mayor población mestiza del globo, el Brasil podrá más fácilmente desempeñar papel de puente entre los continentes.

La política externa brasileña sufrió también varias modificaciones bajo el influjo separado o concomitante de fuerzas endógenas y exógenas. Habría muchos ejemplos. Así, en los años i961-1964 las autoridades de Brasilia se alejaron considerablemente de Washington, reanudaron o ampliaron vínculos con el Este europeo y se volcaron más hacia los países en desarrollo. Aún en el período iniciado con el movimiento de 1964 hasta nuestros dfas ocurrieron cambios importantes ${ }^{61}$. La dimensión tercermundista es enfatizada en los Gobiernos de Costa y Silva, Geisel y Figueiredo. Cabe resaltar que el panorama internacional también evolucionó mucho. El predominio americano-soviético de postguerra fue debilitado por el fortalecimiento y por el dinamismo japonés, por el alejamiento

"Figueiredo, João. Mensagem ao Congresso Nacional. Brasilia, DIN, 1982, página 162.

${ }^{0}$ Mrartins, Carlos Estevam. "A evolução da polf́tica externa brasileira na década de 64/74". Estudos CEBRAP, 12: 53-99, abr./mayo/jun. 1975. 
entre Moscú y Pekín, ampliación de la Comunidad Económica Europea, etc. . ${ }^{62}$.

El notable grado de continuidad de la diplomacia brasileña debe mucho al recuerdo permanente de su figura clave, el Barón de Rio Branco (1845-1912). José Maria da Silva Paranhos Junior tuvo como su primer puesto Liverpool, en el exterior, en 1876. Alli intensificó sus estudios de Historia, particularmente importantes para el conocimiento de la realidad nacional. De 1902 a 1912, año de su fallecimiento, ocupó el cargo de Canciller. Se distinguió notablemente en las negociaciones concernientes a la fijación de las fronteras, tarea necesaria y compleja, sobre todo en el caso de un país con 10 vecinos. En gran parte gracias a él, Brasil no tiene litigios de límites.

En Río de Janeiro, el Ministerio de Relaciones Exteriores funcionó durante mucho tiempo en la antigua mansión del Barón, el Palacio Itamaraty. El moderno edificio donde trabajan en Brasilia los diplomáticos brasileños, es conocido como Palacio Itamaraty. Sus grandes dimensiones y su proximidad a las sedes de los Tres Poderes parecen testimoniar la importancia particular atribuida por el país a Ia política externa. Fundado en 1945, el Instituto encargado de reclutar y formar a los futuros diplomáticos ostenta el nombre de Rio Branco. La orden Rio Branco reúne personas destacadas por servicios prestados en el área internacional. La publicación de las obras de Rio Branco, la evocación frecuente de sus enseñanzas y de su vicla de trabajo y dedicación al país, son así inspiración perenne de la política externa del país.

Escribió el Canciller Saraiva Guerreiro: "No afectamos el destino del Sistema Internacional en la misma forma amplia por la cual éste configura lo cotidiano de la vida brasileña. Se trata de un problema estructural: nuestra dependencia de la importación de petróleo, tecnologfa y capitales". O Brasil e sua Politica Externa. Brasília, UnB. 1981, p. 5. 


\section{BIBLIOGRAFIA CITADA}

Ardao, Arturo. Génesis de la idea y' el nombre de América Latina. Caracas. Centro de Estudios Latinoamericanos Rómulo Gallegos. I980.

BAstrde, Roger. Le Prochain et le Lointain. Paris. Editions Cujas, 1970.

Castello Branco, H. A. de. Discutsos de 1964, Brasilia, Secretaria, de Imprensa, s.d.

Casrro, Therezinha de. Africa Geohistória, Geopolitica e Relações Internacionais. Rio de Janeiro, Biblioteca do Exército Editora, 1981.

El procieso de Integración en América Latina. Buenos Aires INTAL-BID, 1982.

Fest, Joachim. Hitler. Rio de Janeiro, Editora Nova Fronteira, 1976.

Figueiredo, João. Mensagem ao Congresso Nacional, Brasília, Departamento de Imprensa Nacional, 1982.

Frguerredo, João. "Discurso na Asem. bléia Geral da onu". Estado de São Paulo, 28/9/1982, p. 6.

Franco, Alvaro da Costa. "O Brasil e a atual conjutura Econômica Mundial": Revista Brasileira de Estudos Políticos, No 47; 195-211 jul. 1978.

Fulbricht, James. As Perspectivas do Ocidente. Rio de Janeiro, Ed. Record, 1964.

Garaudy, Roger. O Ocidente é um Acidente, Rio de Janeiro, Salamandra, 1978.

Guerretro, Ramiro Saraiva. "Política Externa do Brasil": Segurança e Desenvolvimento. $\mathrm{xxx}$ (I90) 42-43, 1982.

GUERREIRo, Ramiro Saraiva. Entrevista. Jornal do Brasil, 13/9/81.

Guerrerro, Ramiro Saraiva. "O Brasil e sua Politica Externa". Brasília, 1981.

GUERREIRo, Ramiro Saraiva. "Os con. trovertidos resultados de Cancún". Folha de São Paulo, 15/11/81.

Guerreiro, Ramiro Saraiva. "Diálogo de confiança e Amizade". Afrochamber, jan. fev. 1982, pp. 4-7.

GUERREIRo, Ramiro Saraiva. Palestra do Chanceler en Londres, no Real
Instituto de Relaçöes Internacio. nais (Chattam House). Texto mimeo.

Guerreiro, Ramiro Saraiva. "A Política Exterior do Brasil". Conferência na Escola Superior de Guerra. Rio de Janeiro, 3/9/1982. Texto datilografaclo.

"Itamaraty divulga programa de Guerreiro no exterior". Jornal de Brasilia, 9/10/1982, p. 14.

LeBrer, Louis Joseph. Suicidio ou Sobrevivência do Ocidente, problemas Fundamentais de nossa Civili. zação. São Paulo, Duas Cidades, 1964.

Lima, Nestor dos Santos. La Imagen del Brasil en las Cartas de Bolivar. Rio de Janeiro, Banco do Brasil, s. d.

Lrma, Paulo Tarso Flecha de. "Comércio Exterior do Brasil. Participação do Itamaraty no Processo de Promoção das Exportações". $R e^{-}$ vista Brasileira de Estudos Politi. cos No 54: 19-39. jan., 1982.

Lima, Paulo Tarso Flecha de. (Entrevista). Visão $\mathrm{xxx}(50): 29-32,14 /$ $12 / 1981$.

Marias, Julián. "O Ocidente e seus Inimigos". Visão, 14/9/81, pp. 70. 72.

MArias, Julián. "As Ameaças ao Ocidente", Estado de São Paulo, Caderno de Cultura. 15/11/1981, pp. 5-7.

Martins, Carlos Estevam. "A evolução de polftica externa brasileira na década 64/74". Estudos GEBRAP No 12-53-99. abr. mai. jun. 1965.

Neto, Leonardo Mota. "Diálogo Brasil e Estados Unidos". Jornal de Brasilia. $26 / 9 / 82$, p. 8 .

PeNa, João Gamilo. Introduction to Bsiazil, Grangula Editions, I Estocolmo. Embaixada do Brasil. 1982.

Penna, J. O. de Meira. "Oriente e Ocidente". Revista Brasileira de Estudos Politicos, No 19: 7-59, jun. 1965.

SARDENBERG, Ronaldo Mota. "A Polftica Externa do Brasil nas últimas décadas". Revista do Serviçõ Públi- 
co, Brasília, vol. 109, $38(4)$, out. dez, 1981.

Selcher, Wayne. Brazil's Multilateral Relalions. Boulder, Westview Press, 1978.

SELGHER, Wayne. Brazil in the World: Brazil in the International System:

The Rise of a Middle Power. Edited by Wayne Selcher, Boulder, Colorado, Westview Press, 1981.

Silva, Golbery do Couto. Geopolitica Brasil. Rio de Janeiro, José Olympio, 1967.

SilverA, Antonio Azeredo da "Bases da Diplomacia Brasileira". Discursos perante as Comissões de Relações Exteriores do Senado Federal e da Câmara dos Deputados. Resenha de Politica Exterior, Brasilia, Minis- tério da Relações Exteriores, No 10: 57-67, jul. ago. set. 1976.

SPENGler, Oswald. A Decadência do Ocidente. Rio de Janeiro, Zahar, 1973.

TAmFr, Alberto. "Brasil, Crescimento e Dependência". Estado de São Paulo, 5/12/81, p. 145.

ToYNBEe, Arnold. Estudos de História Contemporânea, A Civilização posta a prova. O Mundo Ocidente. São Paulo, Campanhia Editora, Nacional, 1976.

Trindade, Antônio Augusto Cançado. "Posições Internacionais do Brasil no Plano Multilateral". Revista Brasileira de Estudos Políticos. No 52: 147-219, Jan. 1981 